\title{
Packing Constant in Orlicz Sequence Spaces Equipped with the p-Amemiya Norm
}

\author{
Xin He, ${ }^{1,2}$ Jijie Yu, ${ }^{3}$ Yunan Cui, ${ }^{4}$ and Xin $\mathrm{Huo}^{5}$ \\ ${ }^{1}$ Department of Mathematics, Harbin Institute of Technology, Harbin 150001, China \\ ${ }^{2}$ Department of Mathematics, Harbin Normal University, Harbin 150025, China \\ ${ }^{3}$ Department of Mathematics, Harbin Electric Power Vocational Technology College, Harbin 150001, China \\ ${ }^{4}$ Department of Mathematics, Harbin University of Science and Technology, Harbin 150080, China \\ ${ }^{5}$ Control and Simulation Center, Harbin Institute of Technology, Harbin 150080, China
}

Correspondence should be addressed to Xin He; hexin8323@163.com

Received 15 January 2014; Accepted 10 May 2014; Published 10 June 2014

Academic Editor: Vladimir Danilov

Copyright (c) 2014 Xin He et al. This is an open access article distributed under the Creative Commons Attribution License, which permits unrestricted use, distribution, and reproduction in any medium, provided the original work is properly cited.

The problem of packing spheres in Orlicz sequence space $l_{\Phi, p}$ equipped with the p-Amemiya norm is studied, and a geometric characteristic about the reflexivity of $l_{\Phi, p}$ is obtained, which contains the relevant work about $l^{p}(p>1)$ and classical Orlicz spaces $l_{\Phi}$ discussed by Rankin, Burlak, and Cleaver. Moreover the packing constant as well as Kottman constant in this kind of spaces is calculated.

\section{Introduction and Preliminaries}

The packing constant is an important and interesting geometric parameter for studying the geometric structure, isometric embedding, noncompactness, and reflexivity in Banach spaces [1-4]. Let $X$ be a Banach space. We denote by $B(X)$ the unit ball of $X$ and by $S(X)$ the unit sphere of $X$. The packing constant $P(X)$ of $X$ is the real number such that if $r \leq P(X)$, then an infinite number of spheres of radius $r$ can be packed in $B(X)$, and if $r>P(X)$, only a finite number of spheres can be done. It began in the 1950s studying the packing constant of special sequence spaces. Burlak et al. [1] proved that $P\left(l^{1}\right)=$ $P\left(l^{\infty}\right)=1 / 2$ and $P\left(l^{p}\right)=1 /\left(1+2^{1-(1 / p)}\right)$ for $1<p<\infty$. Rankin found $P\left(l^{2}\right)$ and $P\left(l^{P}\right)(p>1)$ in 1955 and 1958, respectively. In 1976, Cleaver discussed Orlicz sequence space $l_{\Phi}^{\circ}$ equipped with the Orlicz norm under a strong condition, and he found upper and lower bounds of $P\left(l_{\Phi}^{\circ}\right)$. In 1983, Ye investigated Orlicz sequence space $l_{\Phi}$ equipped with the Luxemburg norm and obtained a formula for $P\left(l_{\Phi}\right)$ [5].

In this paper, an analogue for Orlicz sequence spaces equipped with the p-Amemiya norm is illustrated, and some useful definitions and lemmas are presented.
Definition 1 (see [1]). The packing constant of a Banach space $X$ is defined by

$$
\begin{gathered}
P(X)=\sup \left\{r>0: \text { there exists }\left\{x_{i}\right\}_{i=1}^{\infty},\left\|x_{i}\right\| \leq 1-r,\right. \\
\left.\left\|x_{i}-x_{j}\right\| \geq 2 \text { for } i, j \in \mathbb{N}, i \neq j\right\} .
\end{gathered}
$$

It is obvious that $P(X)=0$, if $\operatorname{dim} X<\infty$.

Lemma 2 (see [2]). Let $X$ be an infinite-dimensional Banach space. Define

$$
K(X)=\sup \left\{\inf \left\{\left\|x_{n}-x_{m}\right\|: n \neq m\right\}:\left\{x_{n}\right\}_{n=1}^{\infty} \subset S(X)\right\},
$$

which is called the Kottman constant of X. Then

$$
P(X)=\frac{K(X)}{K(X)+2} .
$$

It is known that $1 \leq K(X) \leq 2$. Due to Riesz lemma, it can be summarised that $K(X) \geq 1$ for any infinite-dimensional Banach space $X$. Finite-dimensional spaces have Kottman 
constant equal to zero. Furthermore, Elton and Odell in [6] proved that if $X$ is an infinite-dimensional Banach space, then there exists an $\varepsilon>0$ such that $K(X) \geq 1+\varepsilon$. Consequently, $1 / 3 \leq P(X) \leq 1 / 2$. Hudzik proved that $P(Y)=1 / 2$ and $K(Y)=2$ for every nonreflexive Banach lattice $Y$ [7].

Recall that a Banach space $X$ is said to be $P$-convex (see [2]) if $P(n, X)<1 / 2$, for some $n \in \mathbb{N}, n \geq 2$, where

$$
\begin{gathered}
P(n, X)=\sup \left\{r>0: \text { there exist }\left\{x_{i}\right\}_{i=1}^{n},\left\|x_{i}\right\| \leq 1-r,\right. \\
\left.\left\|x_{i}-x_{j}\right\| \geq 2 r \text { for } i \neq j\right\} .
\end{gathered}
$$

Kottman [2] has proved that any $P$-convex Banach space is reflexive.

The packing problem in Orlicz sequence spaces was investigated in [8-11]. The packing constant for MusielakOrlicz sequence spaces and Cesaro sequence spaces have been calculated in $[12,13]$.

For any map $\Phi: \mathbb{R} \rightarrow[0, \infty]$, define

$$
\begin{aligned}
& a_{\Phi}=\max \{u \geq 0: \Phi(u)=0\}, \\
& b_{\Phi}=\max \{u \geq 0: \Phi(u)<\infty\} .
\end{aligned}
$$

A map $\Phi$ is said to be an Orlicz function, if $\Phi(0)=0$; $\Phi$ is not identically equal to zero; it is even and convex on the interval $\left(-b_{\Phi}, b_{\Phi}\right)$ and left-continuous at $b_{\Phi}$.

For every Orlicz function $\Phi$, we define its complementary function $\Psi: \mathbb{R} \rightarrow[0, \infty]$ by the formula

$$
\Psi(v)=\sup \{u|v|-\Phi(u): u \geq 0\} .
$$

The complementary function $\Psi$ is also an Orlicz function. The convex modular $I_{\Phi}$ is defined on $l^{0}$ (the space of all real sequences) by $I_{\Phi}(x)=\sum_{i=1}^{\infty} \Phi(x(i))$ for any $x=(x(i))$.

Definition 3 (see [14-16]). The Orlicz sequence space is defined as the set

$$
l_{\Phi}=\left\{x=(x(i)): I_{\Phi}(\lambda x)<\infty \text {, for some } \lambda>0\right\} .
$$

The Luxemburg norm and the Orlicz norm are expressed as

$$
\begin{gathered}
\|x\|_{\Phi}=\inf \left\{\lambda>0: I_{\Phi}\left(\frac{x}{\lambda}\right) \leq 1\right\}, \\
\|x\|_{\Phi}^{\circ}=\inf _{k>0} \frac{1}{k}\left(1+I_{\Phi}(k x)\right),
\end{gathered}
$$

respectively. The Orlicz space equipped with the Luxemburg norm and the Orlicz norm are denoted by $l_{\Phi}$ and $l_{\Phi}^{\circ}$, respectively.

For any $1 \leq p \leq \infty$ and $u \geq 0$, define

$$
s_{p}(u)= \begin{cases}\left(1+u^{p}\right)^{1 / p}, & \text { for } 1 \leq p<\infty, \\ \max \{1, u\}, & \text { for } p=\infty\end{cases}
$$

and define $s_{\Phi, p}(x)=s_{p} \circ I_{\Phi}(x)$ for all $1 \leq p \leq \infty$. Note that the functions $s_{p}$ and $s_{\Phi, p}$ are convex. Moreover, the function $s_{p}$ is increasing on $\mathbb{R}_{+}$, for $1 \leq p<\infty$, but the function $s_{\infty}$ is increasing on the interval $[1, \infty)$ only.
Definition 4 (see $[17,18])$. Let $1 \leq p \leq \infty$. For any $x=(x(i))$, define the p-Amemiya norm by the formula

$$
\|x\|_{\Phi, p}=\inf _{k>0} \frac{1}{k} s_{\Phi, p}(k x) .
$$

The Orlicz space equipped with the p-Amemiya norm will be denoted by $l_{\Phi, p}$.

It is known that $\|x\|_{\Phi, 1}=\|x\|_{\Phi}^{\circ}$ and $\|x\|_{\Phi, \infty}=\|x\|_{\Phi}$. If $1 \leq p<\infty, x \neq 0$, then

$$
\frac{1}{2}\|x\|_{\Phi}^{\circ} \leq\|x\|_{\Phi} \leq\|x\|_{\Phi, p} \leq 2^{1 / p}\|x\|_{\Phi}<2^{1 / p}\|x\|_{\Phi}^{\circ} .
$$

(See [17].)

Let $p_{+}$be the right-hand side derivative of $\Phi$ on $\left[0, b_{\Phi}\right)$ and put $p_{+}\left(b_{\Phi}\right)=\lim _{u \rightarrow b_{\Phi}^{-}} p_{+}(u)$. Define the function $\alpha_{p}$ : $l_{\Phi, p} \rightarrow[-1, \infty]$ by

$$
\alpha_{p}(x)= \begin{cases}I_{\Phi}^{p-1}(x) I_{\Psi}\left(p_{+}(|x|)\right)-1, & 1 \leq p<\infty, \\ -1, & p=\infty, I_{\Phi}(x) \leq 1, \\ I_{\Psi}\left(p_{+}(|x|)\right), & p=\infty, I_{\Phi}(x)>1\end{cases}
$$

and the functions $k_{p}^{*}: l_{\Phi, p} \rightarrow[0, \infty)$ and $k_{p}^{* *}: l_{\Phi, p} \rightarrow$ $[0, \infty)$ by

$$
\begin{gathered}
k_{p}^{*}(x)=\inf \left\{k \geq 0: \alpha_{p}(k x) \geq 0\right\}, \quad(\text { with inf } \phi=\infty), \\
k_{p}^{* *}(x)=\inf \left\{k \geq 0: \alpha_{p}(k x) \leq 0\right\} .
\end{gathered}
$$

It is obvious that $k_{p}^{*}(x) \leq k_{p}^{* *}(x)$ for every $1 \leq p \leq \infty$ and $x \in l_{\Phi, p}$.

$$
\text { Set } K_{p}(x)=\left\{0<k<\infty: k_{p}^{*}(x) \leq k \leq k_{p}^{* *}(x)\right\} \text {. }
$$

Definition 5 (see [14]). We say an Orlicz function $\Phi$ satisfies the $\Delta_{2}(0)$-condition ( $\Phi \in \Delta_{2}(0)$, for short) if there exist constants $K \geq 2$ and $u_{0}>0$ such that $\Phi\left(u_{0}\right)>0$ and

$$
\Phi(2 u) \leq K \Phi(u) \quad \text { for every }|u| \leq u_{0} .
$$

For more details about Orlicz spaces, we refer the reader to $[15,16,18,19]$.

Lemma 6 (see [20]). Assume that $\Phi \in \Delta_{2}(0), 1 \leq p<\infty$. Then, for any $L>0$ and $\varepsilon>0$, there exists $\delta>0$ such that for any $x, y \in l^{0}$ there holds the implication

$$
\left(I_{\Phi}(x) \leq L\right) \wedge\left(I_{\Phi}(y) \leq \delta\right) \Longrightarrow\left|I_{\Phi}^{p}(x+y)-I_{\Phi}^{p}(x)\right|<\varepsilon .
$$

\section{Main Results}

Assume that $\Phi \in \Delta_{2}(0), 1 \leq p<\infty$. Then, for any $x \in S\left(l_{\Phi, p}\right)$ and $k>1$, there exists a unique $d_{x, k}>0$ such that

$$
I_{\Phi}^{p}\left(\frac{k x}{d_{x, k}}\right)=\frac{k^{p}-1}{2^{p}} .
$$


Set

$$
\begin{gathered}
d_{x}=\inf \left\{d_{x, k}: k>1\right\}, \\
d=\sup \left\{d_{x}: x \in S\left(l_{\Phi, p}\right)\right\} .
\end{gathered}
$$

Then $d_{x}>1$ and $1<d \leq 2$. Denote

$$
\begin{aligned}
k^{\prime} & =\inf \left\{k: k \in K_{p}(x),\|x\|_{\Phi, p}=1\right\}, \\
k^{\prime \prime} & =\sup \left\{k: k \in K_{p}(x),\|x\|_{\Phi, p}=1\right\} .
\end{aligned}
$$

In the sequel, the packing constant $l_{\Phi, p}$ is calculated, and the main results of this paper are proposed.

Theorem 7. If $\Phi \in \Delta_{2}(0), 1 \leq p<\infty$, then $K\left(l_{\Phi, p}\right)=d$ and $P\left(l_{\Phi, p}\right)=d /(d+2)$.

Proof. For any $\varepsilon>0$, there exists $x \in S\left(l_{\Phi, p}\right)$ such that $d_{x}>$ $d-\varepsilon$, so $d_{x, k}>d-\varepsilon$ for all $k>1$. Define

$$
x^{n}=\sum_{i=1}^{\infty} x(i) e_{2^{n-1}(2 i-1)}, \quad \forall n \in \mathbb{N} .
$$

Then $\left\{x^{n}\right\}$ have pairwise disjoint supports and $\left\|x^{n}\right\|_{\Phi, p}=$ $\|x\|_{\Phi, p}=1(n \in \mathbb{N})$. For all $n \neq m$ and all $k>1$,

$$
\begin{aligned}
\frac{1}{k}( & \left.1+I_{\Phi}^{p}\left(k \frac{x^{n}-x^{m}}{d-\varepsilon}\right)\right)^{1 / p} \\
= & \frac{1}{k}\left(1+2^{p} I_{\Phi}^{p}\left(\frac{k x}{d-\varepsilon}\right)\right)^{1 / p} \\
> & \frac{1}{k}\left(1+2^{p} I_{\Phi}^{p}\left(\frac{k x}{d_{x, k}}\right)\right)^{1 / p} \\
= & \frac{1}{k}\left(1+2^{p} \cdot \frac{k^{p}-1}{2^{p}}\right)^{1 / p}=1 .
\end{aligned}
$$

Then $\left\|x^{n}-x^{m}\right\|_{\Phi, p}=\inf _{k>0}(1 / k)\left(1+I_{\Phi}^{p}\left(k\left(x^{n}-x^{m}\right)\right)\right)^{1 / p} \geq$ $d-\varepsilon$, so we have $K\left(l_{\Phi, p}\right) \geq d$, since $\varepsilon$ is arbitrary.

In the following, $K\left(l_{\Phi, p}\right) \leq d$ will be illustrated as an important part of our results.

For any sequence $\left\{x_{n}\right\} \subset S\left(l_{\Phi, p}\right)$, which means that $x_{n}=$ $\left(x_{n}(i)\right)_{i},\left\|x_{n}\right\|_{\Phi, p}=\left\|\sum_{i=1}^{\infty} x_{n}(i) e_{i}\right\|_{\Phi, p}=1$, for any $n \in \mathbb{N}$, then $\left\{\left\|x_{n}(i) e_{i}\right\|_{\Phi, p}\right\}_{n}$ is bounded for all $i \in \mathbb{N}$.

Since $\left\{\left\|x_{n}(1) e_{1}\right\|_{\Phi, p}\right\}_{n}$ is bounded, there exists a subsequence $\left\{x_{1_{n}}\right\} \subset\left\{x_{n}\right\}$ such that $\left\{\left\|x_{1_{n}}(1) e_{1}\right\|_{\Phi, p_{n}}\right\}$ is convergent, but $\left\{\left\|x_{1_{n}}(2) e_{2}\right\|_{\Phi, p}\right\}$ is bounded, so there exists a subsequence $\left\{x_{2_{n}}\right\} \subset\left\{x_{1_{n}}\right\}$ such that $\left\{\left\|x_{2_{n}}(2) e_{2}\right\|_{\Phi, p}\right\}$ is convergent. In a similar way, using the diagonal method, we can find a subsequence $\left\{x_{n_{n}}\right\} \subset\left\{x_{n}\right\}$ such that, for any $i \in \mathbb{N}$, $\left\{\left\|x_{n_{n}}(i) e_{i}\right\|_{\Phi, p}\right\}_{n \geq i}$ is convergent. Denoting $\left\|e_{i}\right\|_{\Phi, p}=s_{i}$ and setting $\left\|x_{n_{n}}(i) e_{i}\right\|_{\Phi, p} \rightarrow b_{i}$ as $n \rightarrow \infty$, then $\left|x_{n_{n}}(i)\right| \rightarrow b_{i} / s_{i}$ as $n \rightarrow \infty$ for all $i \in \mathbb{N}$.

Let $x=\left(b_{i} / s_{i}\right)_{i},\left|x_{n_{n}}\right|=\left(\left|x_{n_{n}}(i)\right|\right)_{i}$, and $z_{n}=\left|x_{n_{n}}\right|-x$. Then

$$
\begin{aligned}
& z_{n}(i) \longrightarrow 0 \text { as } n \longrightarrow \infty \quad \forall i \in \mathbb{N}, \\
& \operatorname{sep}\left(z_{n}\right)=\operatorname{sep}\left(\left|x_{n_{n}}\right|\right) \geq \operatorname{sep}\left(x_{n}\right) .
\end{aligned}
$$

Since $\Phi \in \Delta_{2}$, then $x \in S\left(l_{\Phi, p}\right)$. For any $\varepsilon>0$, there exists $i_{0} \in \mathbb{N}$, such that $\left\|\sum_{i=i_{0}+1}^{\infty} x(i) e_{i}\right\|_{\Phi, p}<\varepsilon$. Moreover, $\left|x_{n_{n}}(i)\right| \rightarrow$ $x(i)$ as $n \rightarrow \infty$ for $i=1, \ldots, i_{0}$. So we have

$$
\begin{aligned}
\left\|z_{n}\right\|_{\Phi, p}= & \left\|\sum_{i=1}^{\infty}\left(\left|x_{n_{n}}(i)\right|-x(i)\right) e_{i}\right\|_{\Phi, p} \\
\leq & \left\|\sum_{i=1}^{i_{0}}\left(\left|x_{n_{n}}(i)\right|-x(i)\right) e_{i}\right\|_{\Phi, p} \\
& +\left\|\sum_{i=i_{0}+1}^{\infty}\left|x_{n_{n}}(i)\right| e_{i}\right\|_{\Phi, p}+\varepsilon,
\end{aligned}
$$

and, consequently, $\lim \sup _{n}\left\|z_{n}\right\|_{\Phi, p} \leq 1+\varepsilon$.

For the above $\varepsilon>0$, since $l_{\Phi, p}$ is order continuous, there exists $i_{1} \in \mathbb{N}$ such that $\left\|\sum_{i=i_{1}+1}^{\infty} z_{n_{1}}(i) e_{i}\right\|_{\Phi, p}<\varepsilon$ for $n_{1}=1$. Take $n_{2}>n_{1}$ such that $\left\|\sum_{i=1}^{i_{1}} z_{n_{2}}(i) e_{i}\right\|_{\Phi, p}<\varepsilon$. And for $n_{2}$, there exists $i_{2}>i_{1}$ such that $\left\|\sum_{i=i_{2}+1}^{\infty} z_{n_{2}}(i) e_{i}\right\|_{\Phi, p}<\varepsilon$. Then

$$
\begin{aligned}
\operatorname{sep}\left(z_{n}\right) \leq & \left\|z_{n_{1}}-z_{n_{2}}\right\|_{\Phi, p} \\
\leq & \left\|\sum_{i=1}^{i_{1}} z_{n_{1}}(i) e_{i}-\sum_{i=i_{1}+1}^{i_{2}} z_{n_{2}}(i) e_{i}\right\|_{\Phi, p} \\
& +\left\|\sum_{i=i_{1}+1}^{\infty} z_{n_{1}}(i) e_{i}\right\|_{\Phi, p} \\
& +\left\|\sum_{i=1}^{i_{1}} z_{n_{2}}(i) e_{i}\right\|_{\Phi, p}+\left\|\sum_{i=i_{2}+1}^{\infty} z_{n_{2}}(i) e_{i}\right\|_{\Phi, p} \\
\leq & \left\|\sum_{i=1}^{i_{1}} z_{n_{1}}(i) e_{i}-\sum_{i=i_{1}+1}^{i_{2}} z_{n_{2}}(i) e_{i}\right\|_{\Phi, p}+3 \varepsilon .
\end{aligned}
$$

Take $n_{3}>n_{2}$ such that $\left\|\sum_{i=1}^{i_{2}} z_{n_{3}}(i) e_{i}\right\|_{\Phi, p}<\varepsilon$, and for $n_{3}$, there exists $i_{3}>i_{2}$ such that $\left\|\sum_{i=i_{3}+1}^{\infty} z_{n_{3}}(i) e_{i}\right\|_{\Phi, p}<\varepsilon$. Then

$$
\begin{aligned}
\| z_{n_{1}}- & z_{n_{3}} \|_{\Phi, p} \\
\leq & \left\|\sum_{i=1}^{i_{1}} z_{n_{1}}(i) e_{i}-\sum_{i=i_{2}+1}^{i_{3}} z_{n_{3}}(i) e_{i}\right\|_{\Phi, p} \\
& +\left\|\sum_{i=i_{1}+1}^{\infty} z_{n_{1}}(i) e_{i}\right\|_{\Phi, p}+\left\|\sum_{i=1}^{i_{2}} z_{n_{3}}(i) e_{i}\right\|_{\Phi, p} \\
& +\left\|\sum_{i=i_{3}+1}^{\infty} z_{n_{3}}(i) e_{i}\right\|_{\Phi, p}
\end{aligned}
$$




$$
\begin{gathered}
\leq\left\|\sum_{i=1}^{i_{1}} z_{n_{1}}(i) e_{i}-\sum_{i=i_{2}+1}^{i_{3}} z_{n_{3}}(i) e_{i}\right\|_{\Phi, p}+3 \varepsilon, \\
\left\|z_{n_{2}}-z_{n_{3}}\right\|_{\Phi, p} \\
\leq\left\|\sum_{i=i_{1}+1}^{i_{2}} z_{n_{2}}(i) e_{i}-\sum_{i=i_{2}+1}^{i_{3}} z_{n_{3}}(i) e_{i}\right\|_{\Phi, p} \\
\quad+\left\|\sum_{i=1}^{i_{1}} z_{n_{2}}(i) e_{i}\right\|_{\Phi, p}+\left\|\sum_{i=i_{2}+1}^{\infty} z_{n_{2}}(i) e_{i}\right\|_{\Phi, p} \\
\quad+\left\|\sum_{i=1}^{i_{2}} z_{n_{3}}(i) e_{i}\right\|_{\Phi, p}+\left\|\sum_{i=i_{3}+1}^{\infty} z_{n_{3}}(i) e_{i}\right\|_{\Phi, p} \\
\leq\left\|\sum_{i=i_{1}+1}^{i_{2}} z_{n_{2}}(i) e_{i}-\sum_{i=i_{2}+1}^{i_{3}} z_{n_{3}}(i) e_{i}\right\|_{\Phi, p}+4 \varepsilon .
\end{gathered}
$$

Analogously, we can find by induction a subsequence $\left\{z_{n_{k}}\right\}$ of $\left\{z_{n}\right\}$ and $\left\{i_{k}\right\} \subset \mathbb{N}$ such that $n_{1}<n_{2}<\cdots<n_{k}<\cdots$, $i_{1}<i_{2}<\cdots<i_{k}<\cdots$ such that, for any $k \in \mathbb{N}$,

$$
\begin{gathered}
\left\|\sum_{i=1}^{i_{k-1}} z_{n_{k}}(i) e_{i}\right\|_{\Phi, p}<\varepsilon, \\
\left\|\sum_{i=i_{k}+1}^{\infty} z_{n_{k}}(i) e_{i}\right\|_{\Phi, p}<\varepsilon, \\
\left\|z_{n_{1}}-z_{n_{k}}\right\|_{\Phi, p} \leq\left\|\sum_{i=1}^{i_{1}} z_{n_{1}}(i) e_{i}-\sum_{i=i_{k-1}+1}^{i_{k}} z_{n_{k}}(i) e_{i}\right\|_{\Phi, p}+3 \varepsilon, \\
\left\|z_{n_{l}}-z_{n_{k}}\right\|_{\Phi, p} \leq\left\|\sum_{i=i_{l-1}+1}^{i_{l}} z_{n_{l}}(i) e_{i}-\sum_{i=i_{k-1}+1}^{i_{k}} z_{n_{k}}(i) e_{i}\right\|_{\Phi, p}+4 \varepsilon, \\
\forall 1<l<k .
\end{gathered}
$$

Since $\left\|\sum_{i=1}^{\infty} z_{n}(i) e_{i}\right\|_{\Phi, p} \leq 1+\varepsilon,\left\|\sum_{i=i_{k-1}+1}^{i_{k}} z_{n_{k}}(i) e_{i}\right\|_{\Phi, p} /(1+\varepsilon) \leq$ 1 for all $k \in \mathbb{N}$. Therefore, for any $l, k \in \mathbb{N}$,

$$
\begin{aligned}
\| z_{n_{l}} & -z_{n_{k}} \|_{\Phi, p} \\
& \leq(1+\varepsilon) \\
& \quad \times\left\|\frac{\sum_{i=i_{l-1}+1}^{i_{l}} z_{n_{l}}(i) e_{i}}{\left\|\sum_{i=i_{l-1}+1}^{i_{1}} z_{n_{l}}(i) e_{i}\right\|_{\Phi, p}}-\frac{\sum_{i=i_{k-1}+1}^{i_{k}} z_{n_{k}}(i) e_{i}}{\left\|\sum_{i=i_{k-1}+1}^{i_{k}} z_{n_{k}}(i) e_{i}\right\|_{\Phi, p}}\right\|_{\Phi, p}
\end{aligned}
$$$$
+4 \varepsilon \text {. }
$$

Setting $y_{k}=\sum_{i=i_{k-1}+1}^{i_{k}} z_{n_{k}}(i) e_{i} /\left\|\sum_{i=i_{k-1}+1}^{i_{k}} z_{n_{k}}(i) e_{i}\right\|_{\Phi, p}$ (for all $k \in \mathbb{N})$, then

$$
\left\{y_{m}\right\} \subset S\left(l_{\Phi, p}\right), \quad \operatorname{supp}\left(y_{l}\right) \cap \operatorname{supp}\left(y_{m}\right)=\phi .
$$

In this way, we get

$$
\begin{aligned}
K\left(l_{\Phi, p}\right) & \leq \operatorname{sep}\left(x_{n}\right) \leq \operatorname{sep}\left(z_{n}\right) \leq \operatorname{sep}\left(z_{n_{i}}\right) \\
& \leq(1+\varepsilon)\left\|y_{m}-y_{l}\right\|_{\Phi, p}+4 \varepsilon .
\end{aligned}
$$

For any $\varepsilon>0$, by the definition of $d$, there exists $k_{m}>1$ such that $d_{y_{m}, k_{m}}<d+\varepsilon$, where $d_{y_{m}, k_{m}}$ satisfies the equality $I_{\Phi}^{p}\left(k_{m} y_{m} / d_{y_{m}, k_{m}}\right)=\left(k_{m}^{p}-1\right) / 2^{p} \quad(m \in \mathbb{N})$.

Setting $\left\|y_{m}-y_{l}\right\|=\lambda_{m l}$ and taking $k_{m l} \in K_{p}\left(\left(y_{m}-y_{l}\right) / \lambda\right)$, we have

$$
\begin{aligned}
1 & =\left\|\frac{y_{m}-y_{l}}{\lambda_{m l}}\right\|_{\Phi, p} \\
& =\frac{1}{k_{m l}}\left(1+I_{\Phi}^{p}\left(k_{m l}\left(\frac{y_{m}-y_{l}}{\lambda_{m l}}\right)\right)\right)^{1 / p} \\
& =\frac{1}{k_{m l}}\left(1+\left(I_{\Phi}\left(k_{m l}\left(\frac{y_{m}}{\lambda_{m l}}\right)\right)+I_{\Phi}\left(k_{m l}\left(\frac{y_{l}}{\lambda_{m l}}\right)\right)\right)^{p}\right)^{1 / p} .
\end{aligned}
$$

Then

$$
\left(k_{m l}^{p}-1\right)^{1 / p}=I_{\Phi}\left(k_{m l}\left(\frac{y_{m}}{\lambda_{m l}}\right)\right)+I_{\Phi}\left(k_{m l}\left(\frac{y_{l}}{\lambda_{m l}}\right)\right) .
$$

Now we obtain that $\lambda_{m l} \leq \max \left\{d_{y_{m}, k_{m l}}, d_{y_{l}, k_{m l}}\right\}$. If not, $\lambda_{m l}>$ $\max \left\{d_{y_{m}, k_{m l}}, d_{y_{l}, k_{m l}}\right\}$, we have

$$
\begin{gathered}
I_{\Phi}^{p}\left(k_{m l}\left(\frac{y_{m}}{\lambda_{m l}}\right)\right)<\frac{k_{m l}^{p}-1}{2^{p}}, \\
I_{\Phi}^{p}\left(k_{m l}\left(\frac{y_{l}}{\lambda_{m l}}\right)\right)<\frac{k_{m l}^{p}-1}{2^{p}},
\end{gathered}
$$

whence

$$
\begin{aligned}
& \left(I_{\Phi}\left(k_{m l}\left(\frac{y_{m}}{\lambda_{m l}}\right)\right)+I_{\Phi}\left(k_{m l}\left(\frac{y_{l}}{\lambda_{m l}}\right)\right)\right)^{p} \\
& <\left(\left(\frac{k_{m l}^{p}-1}{2^{p}}\right)^{1 / p}+\left(\frac{k_{m l}^{p}-1}{2^{p}}\right)^{1 / p}\right)^{p} \\
& \quad=k_{m l}^{p}-1 .
\end{aligned}
$$

This is a contradiction. Hence,

$$
\left\|y_{m}-y_{l}\right\|_{\Phi, p}=\lambda_{m l} \leq \max \left\{d_{y_{m}, k_{m l}}, d_{y_{l}, k_{m l}}\right\} \leq d .
$$

So $K\left(l_{\Phi, p}\right) \leq(1+\varepsilon) d+4 \varepsilon$; we get $K\left(l_{\Phi, p}\right) \leq d$ due to the arbitrariness of $\varepsilon$.

Theorem 8. If $\Phi \notin \Delta_{2}(0), 1 \leq p<\infty$, then $K\left(l_{\Phi, p}\right)=2$.

Proof. Denote

$$
\begin{aligned}
& l_{\alpha}=\left\{x \in l_{\Phi, p}: \lim _{n \rightarrow \infty}\right. \|(0, \ldots, 0, x(n+1), \\
&\left.x(n+2), \ldots) \|_{\Phi, p}=0\right\} .
\end{aligned}
$$


Since $\Phi \notin \Delta_{2}(0)$, then $l_{\alpha} \neq l_{\Phi, p}$; so for $\varepsilon>0$, according to Riesz lemma, there exists $x_{\varepsilon} \in S\left(l_{\Phi, p}\right)$ satisfying $\operatorname{dist}\left(x_{\varepsilon}, l_{\alpha}\right)>$ $1-\varepsilon$. Then we have

$$
\left\|\left(0, \ldots, 0, x_{\varepsilon}(n+1), x_{\varepsilon}(n+2), \ldots\right)\right\|_{\Phi, p}>1-\varepsilon .
$$

Since

$$
\lim _{m \rightarrow \infty}\left\|\left(0, \ldots, 0, x_{\varepsilon}(n+1), \ldots, x_{\varepsilon}(m), 0, \ldots\right)\right\|_{\Phi, p}>1-\varepsilon,
$$

there exists a subsequence $\left\{n_{i}\right\} \subset \mathbb{N}$ such that $n_{1}<n_{2}<\cdots<$ $n_{k}<\cdots$ and

$$
\left\|\left(0, \ldots, 0, x_{\varepsilon}\left(n_{i}+1\right), \ldots, x_{\varepsilon}\left(n_{i+1}\right), 0, \ldots\right)\right\|_{\Phi, p}>1-\varepsilon .
$$

Let

$$
\begin{aligned}
x_{1}= & \left(-x_{\varepsilon}(1), \ldots,-x_{\varepsilon}\left(n_{1}\right), x_{\varepsilon}\left(n_{1}+1\right), \ldots,\right. \\
& \left.x_{\varepsilon}\left(n_{2}\right), x_{\varepsilon}\left(n_{2}+1\right), \ldots\right), \\
x_{2}= & \left(x_{\varepsilon}(1), \ldots, x_{\varepsilon}\left(n_{1}\right),-x_{\varepsilon}\left(n_{1}+1\right), \ldots,\right. \\
& \left.-x_{\varepsilon}\left(n_{2}\right), x_{\varepsilon}\left(n_{2}+1\right), \ldots\right) .
\end{aligned}
$$

Then for any $m, l \in \mathbb{N}$,

$$
\begin{aligned}
& \left\|x_{m}-x_{l}\right\|_{\Phi, p} \\
& =2 \|\left(\ldots, 0, x_{\varepsilon}\left(n_{m-1}+1\right), \ldots, x_{\varepsilon}\left(n_{m}\right), 0, \ldots,\right. \\
& \left.\quad 0, x_{\varepsilon}\left(n_{l-1}+1\right), \ldots, x_{\varepsilon}\left(n_{l}\right), 0, \ldots\right) \|_{\Phi, p} \\
& \geq 2\left\|\left(0, \ldots, 0, x_{\varepsilon}\left(n_{m-1}+1\right), \ldots, x_{\varepsilon}\left(n_{m}\right), 0, \ldots\right)\right\|_{\Phi, p} \\
& \geq 2(1-\varepsilon) .
\end{aligned}
$$

Due to the arbitrariness of $\varepsilon>0$, we have $K\left(l_{\Phi, p}\right)=2$.

Lemma 9. If $\Phi \in \Delta_{2}(0) \cap \nabla_{2}(0), 1 \leq p<\infty$, then

$$
1<k^{\prime} \leq k^{\prime \prime}<\infty \text {. }
$$

Proof. (1) Since $\Phi \in \Delta_{2}(0)$ and the norm convergence and the modular convergence are equivalent, there exists $c>0$ such that

$$
\inf _{\|x\|_{\Phi, p}=1} I_{\Phi}(x)=c>0 .
$$

For any $x \in S\left(l_{\Phi, p}\right)$ and $k \in K_{p}(x)$, we have

$$
1=\|x\|_{\Phi, p}=\frac{1}{k}\left(1+I_{\Phi}^{p}(k x)\right)^{1 / p}
$$

so $k=\left(1+I_{\Phi}^{p}(k x)\right)^{1 / p} \geq 1$; then

$$
\begin{aligned}
k^{\prime} & =\inf _{\|x\|_{\Phi, p}=1} k=\inf _{\|x\|_{\Phi, p}=1}\left(1+I_{\Phi}^{p}(k x)\right)^{1 / p} \\
& \geq \inf _{\|x\|_{\Phi, p}=1}\left(1+I_{\Phi}^{p}(x)\right)^{1 / p} \geq\left(1+c^{p}\right)^{1 / p}>1 .
\end{aligned}
$$

(2) If $\Phi \in \nabla_{2}(0)$, then there exists $\alpha>1$ such that

$$
u p_{+}(u) \geq \alpha \Phi(u), \quad\left(|u| \leq q_{+}\left(\Psi^{-1}\left(\frac{1}{c^{p-1}}\right)\right)\right) .
$$

For any $x \in S\left(l_{\Phi, p}\right)$ and $k \in K_{p}(x)$, we have $1<k^{\prime} \leq k \leq$ $k_{p}^{* *}(x)$; then for any $\varepsilon \in\left(0, k^{\prime}-1\right)$, we get

$$
\begin{aligned}
1 & \geq I_{\Phi}^{p-1}((k-\varepsilon) x) I_{\Psi}\left(p_{+}(|(k-\varepsilon) x|)\right) \\
& \geq I_{\Phi}^{p-1}(x) I_{\Psi}\left(p_{+}(|(k-\varepsilon) x|)\right) \\
& \geq c^{p-1} \sum_{i=1}^{\infty} \Psi\left(p_{+}(|(k-\varepsilon) x(i)|)\right) \\
& \geq c^{p-1} \Psi\left(p_{+}(|(k-\varepsilon) x(i)|)\right), \quad(\forall i=1,2, \ldots) ;
\end{aligned}
$$

whence $|(k-\varepsilon) x(i)| \leq q_{+}\left(\Psi^{-1}\left(1 / c^{p-1}\right)\right)$. Moreover, according to the Young inequality

$$
\begin{aligned}
& 1 \geq I_{\Phi}^{p-1}((k-\varepsilon) x) I_{\Psi}\left(p_{+}(|(k-\varepsilon) x|)\right) \\
& \geq c^{p-1} \sum_{i=1}^{\infty} \Psi\left(p_{+}(|(k-\varepsilon) x(i)|)\right) \\
& \geq c^{p-1} \sum_{i=1}^{\infty}\left\{|(k-\varepsilon) x(i)| p_{+}(|(k-\varepsilon) x(i)|)\right. \\
& \quad-\Phi((k-\varepsilon) x(i))\} \\
& \geq c^{p-1}(\alpha-1) I_{\Phi}((k-\varepsilon) x) \\
& \geq c^{p-1}(\alpha-1)(k-\varepsilon) I_{\Phi}(x) \\
& c^{p}(\alpha-1)(k-\varepsilon),
\end{aligned}
$$

since $\varepsilon>0$ is arbitrary, we deduce that $k \leq 1 /(\alpha-1) c^{p}<$ $\infty$.

Ye et al. [21] have proved that Orlicz function space as well as Orlicz sequence space equipped with the Luxemburg norm is $P$-convex if and only if it is reflexive; that is, $\Phi$ satisfies the suitable $\Delta_{2}$-condition and $\nabla_{2}$-condition (i.e., the $\Delta_{2}$-condition at zero in the sequence case). We will prove now an analogous result for $l_{\Phi, p}$ in terms of $P\left(l_{\Phi, p}\right)$.

Theorem 10. If $\Phi \in \Delta_{2}(0) \cap \nabla_{2}(0), 1 \leq p<\infty$, then $P\left(l_{\Phi, p}\right)<$ $1 / 2$.

Proof. If $\Phi \in \Delta_{2}(0)$, then due to Lemma 6, for any $\varepsilon>0$, there exists $\delta>0$ such that

$$
\begin{aligned}
& \left(I_{\Phi}(x) \leq 1\right) \wedge\left(I_{\Phi}(y)<\frac{\delta k^{\prime \prime}}{2(2-\delta)}\right) \\
& \quad \Longrightarrow I_{\Phi}^{p}(x+y) \leq I_{\Phi}^{p}(x)+\varepsilon\left(\left(k^{\prime}\right)^{p}-1\right),
\end{aligned}
$$


where $k^{\prime \prime}=\sup \left\{k: k \in K_{p}(x),\|x\|_{\phi, p}=1\right\}$. According to Lemma $9, k^{\prime \prime}<\infty$. Set inf $\tilde{\| x}_{\Phi, p}=1 I_{\Phi}(x)=c>0$. If $K\left(l_{\Phi, p}\right)=$ 2 , then there exists $x \in S\left(l_{\Phi, p}\right)$ such that $d_{x}>2-\delta$, so $d_{x, k}>$ $2-\delta$ for all $k \in K_{p}(x)$.

Since $\in \nabla_{2}(0)$, we can find $\theta>1$ such that

$$
\Phi\left(\frac{u}{2}\right) \leq \frac{1}{2 \theta} \Phi(u), \quad\left(|u| \leq q_{+}\left(\Psi^{-1}\left(\frac{1}{c^{p-1}}\right)\right)\right) .
$$

Let us notice that

$$
I_{\Phi}\left(\frac{\delta k}{2(2-\delta)} x\right) \leq\left\|\frac{\delta k}{2(2-\delta)} x\right\|_{\Phi, p}=\frac{\delta k}{2(2-\delta)} \leq \frac{\delta k^{\prime \prime}}{2(2-\delta)} .
$$

Thus,

$$
\begin{aligned}
\frac{k^{p}-1}{2^{p}} & =I_{\Phi}^{p}\left(\frac{k x}{d_{x, k}}\right)<I_{\Phi}^{p}\left(\frac{k x}{2-\delta}\right) \\
& =I_{\Phi}^{p}\left(\frac{k x}{2}+\frac{\delta k x}{2(2-\delta)}\right) \\
& \leq I_{\Phi}^{p}\left(\frac{k x}{2}\right)+\varepsilon\left(\left(k^{\prime}\right)^{p}-1\right) \\
& \leq \frac{1}{(2 \theta)^{p}} I_{\Phi}^{p}(k x)+\varepsilon\left(k^{p}-1\right) \\
& =\frac{1}{(2 \theta)^{p}}\left(k^{p}-1\right)+\varepsilon\left(k^{p}-1\right) \\
& =\left(\frac{1}{(2 \theta)^{p}}+\varepsilon\right)\left(k^{p}-1\right) ;
\end{aligned}
$$

we have $1 / 2^{p} \leq 1 /(2 \theta)^{p}+\varepsilon$. Since $\varepsilon$ is arbitrary, we obtain $\theta<$ 1 ; this is a contradiction. Therefore, $K\left(l_{\Phi, p}\right)<2$ and $P\left(l_{\Phi, p}\right)<$ $1 / 2$.

Corollary 11. If $X=l^{p_{1}}\left(1<p_{1}<\infty\right)$, then

$$
K\left(l^{p_{1}}\right)=2^{1 / p_{1}}, \quad P\left(l^{p_{1}}\right)=\frac{1}{1+2^{1-\left(1 / p_{1}\right)}} .
$$

Proof. For any $x \in l^{p_{1}}$,

$$
\begin{aligned}
\|x\|_{\Phi, p} & =\left(p_{1}-1\right)^{-1 / p q_{1}} p_{1}^{1 / p-1 / p_{1}}\|x\|_{l^{p_{1}}} \\
& =\left(p_{1}-1\right)^{-1 / p q_{1}} p_{1}^{1 / p-1 / p_{1}} \Phi^{-1}\left(I_{\Phi}(x)\right),
\end{aligned}
$$

where $\Phi(u)=|u|^{p_{1}} / p_{1}$ and $1 / p+1 / q=1,1 / p_{1}+1 / q_{1}=1$.

In fact, since $\Phi(u)=|u|^{p_{1}} / p_{1}$, then $\Phi\left(\|x\|_{p^{p_{1}}}\right)=I_{\Phi}(x)$ and $\Phi^{-1}(u)=\left(p_{1} u\right)^{1 / p_{1}}$. Set

$$
f(k)=\frac{1}{k^{p}}\left(1+I_{\Phi}^{p}(k x)\right)=\frac{1}{k^{p}}\left(1+\left(\frac{k^{p_{1}}\|x\|_{l^{p}}^{p_{1}}}{p_{1}}\right)^{p}\right) .
$$

By $f^{\prime}(k)=0$, we get $k_{0}=\left(p_{1}-1\right)^{-1 / p p_{1}} p_{1}^{1 / p_{1}} 1 /\|x\|_{p^{p_{1}}}$. Since $f^{\prime \prime}\left(k_{0}\right)<0$, we have

$$
\begin{aligned}
\|x\|_{\Phi, p} & =\inf _{k>0} \frac{1}{k}\left(1+I_{\Phi}^{p}(k x)\right)^{1 / p}=\left(f\left(k_{0}\right)\right)^{1 / p} \\
& =\left(p_{1}-1\right)^{-1 / p q_{1}} p_{1}^{1 / p-1 / p_{1}}\|x\|_{l^{p_{1}}} .
\end{aligned}
$$

Set $\alpha=\left(p_{1}-1\right)^{-1 / p q_{1}} p_{1}^{1 / p-1 / p_{1}}$. From the equation

$$
\frac{k^{p}-1}{2^{p}}=I_{\Phi}^{p}\left(\frac{k x}{d_{x, k}}\right)=\Phi^{p}\left(\frac{1}{\alpha}\left\|\frac{k x}{d_{x, k}}\right\|_{\Phi, p}\right)=\Phi^{p}\left(\frac{k}{\alpha d_{x, k}}\right),
$$

we deduce that $d_{x, k}=(k / \alpha)\left(\Phi^{-1}\left(\left(\left(k^{p}-1\right) / 2^{p}\right)^{1 / p}\right)\right)^{-1}$. Therefore,

$$
\begin{aligned}
K\left(l^{p_{1}}\right) & =d=\sup _{\|x\|_{\Phi, p}=1} \inf _{k>1} d_{x, k} \\
& =\frac{1}{\alpha} \inf _{k>1}\left\{k\left(\Phi^{-1}\left(\left(\frac{k^{p}-1}{2^{p}}\right)^{1 / p}\right)\right)^{-1}\right\} \\
& =\left(p_{1}-1\right)^{1 / p q_{1}} p_{1}^{-1 / p} 2^{1 / p_{1}} \inf _{k>1} \frac{k}{\left(k^{p}-1\right)^{1 / p p_{1}}} \\
& =2^{1 / p_{1}} .
\end{aligned}
$$

We have $K\left(l^{p_{1}}\right)=2^{1 / p_{1}}$. So $P\left(l^{p_{1}}\right)=1 /(1+2)^{1-\left(1 / p_{1}\right)}$ for $1<$ $p_{1}<\infty$.

\section{Conflict of Interests}

The authors declare that there is no conflict of interests regarding the publication of this paper.

\section{Acknowledgments}

This work was supported by the Provincial Education Department Fund (12531185) and supported by the National Natural Science Foundation of China (61203191).

\section{References}

[1] J. A. Burlak, R. A. Rankin, and A. P. Robertson, "The packing of spheres in the space $l_{p}$," vol. 4, no. 1, pp. 22-25, 1958.

[2] C. A. Kottman, "Packing and reflexivity in Banach spaces," Transactions of the American Mathematical Society, vol. 150, pp. 565-576, 1970.

[3] J. R. Webb and W. Zhao, "On connections between set and ball measures of noncompactness," The Bulletin of the London Mathematical Society, vol. 22, no. 5, pp. 471-477, 1990.

[4] R. A. Rankin, "On packings of spheres in Hilbert space," Proceedings of the Glasgow Mathematical Association, vol. 2, pp. 145-146, 1955.

[5] Y. N. Ye, "Packing spheres in Orlicz sequence spaces," Chinese Annals of Mathematics B, vol. 4, no. 4, pp. 487-493, 1983. 
[6] J. Elton and E. Odell, "The unit ball of every infinitedimensional normed linear space contains a $(1+\varepsilon)$-separated sequence," Colloquium Mathematicum, vol. 44, no. 1, pp. 105$109,1981$.

[7] H. Hudzik, "Every nonreflexive Banach lattice has the packing constant equal to 1/2," Collectanea Mathematica, vol. 44, no. 13, pp. 129-134, 1993.

[8] C. E. Cleaver, "Packing spheres in Orlicz spaces," Pacific Journal of Mathematics, vol. 65, no. 2, pp. 325-335, 1976.

[9] T. F. Wang, "Packing constants of Orlicz sequence spaces," Chinese Annals of Mathematics A, vol. 8, no. 4, pp. 508-513, 1987.

[10] T. F. Wang and Y. M. Liu, "Packing constant of a type of sequence spaces," Commentationes Mathematicae Prace Matematyczne, vol. 30, no. 1, pp. 197-203, 1990.

[11] H. Hudzik and T. Landes, "Packing constant in Orlicz spaces equipped with the Luxemburg norm," Bollettino della Unione Matematica Italiana A, vol. 9, no. 2, pp. 225-237, 1995.

[12] H. Hudzik, C. X. Wu, and Y. N. Ye, "Packing constant in Musielak-Orlicz sequence spaces equipped with the Luxemburg norm," Revista Matemática de la Universidad Complutense de Madrid, vol. 7, no. 1, pp. 13-26, 1994.

[13] Y. A. Cui and H. Hudzik, "Packing constant for Cesaro sequence space," Nonlinear Analysis: Theory, Methods \& Applications, vol. 47, no. 4, pp. 2695-2702, 2001.

[14] S. T. Chen, "Geometry of Orlicz spaces," Dissertationes Mathematicae, vol. 356, p. 204, 1996.

[15] L. Maligranda, Orlicz Spaces and Interpolation, vol. 5 of Seminars in Mathematics, Universidade Estadual de Campinas, Departamento de Matemática, Campinas, Brazil, 1989.

[16] J. Musielak, Orlicz Spaces and Modular Spaces, vol. 1034 of Lecture Notes in Mathematics, Springer, Berlin, Germany, 1983.

[17] Y. A. Cui, L. F. Duan, H. Hudzik, and M. Wisla, "Basic theory of $p$-Amemiya norm in Orlicz spaces $(1 \leq p \leq \infty)$ : extreme points and rotundity in Orlicz spaces endowed with these norms," Nonlinear Analysis: Theory, Methods \& Applications, vol. 69, no. 5-6, pp. 1796-1816, 2008.

[18] Y. A. Cui, H. Hudzik, J. J. Li, and M. Wisla, "Strongly extreme points in Orlicz spaces equipped with the p-Amemiya norm," Nonlinear Analysis: Theory, Methods \& Applications, vol. 71, no. 12, pp. 6343-6364, 2009.

[19] Y. A. Cui, H. Hudzik, M. Wisla, and K. Wlazlak, "Nonsquareness properties of Orlicz spaces equipped with the $p$ Amemiya norm," Nonlinear Analysis: Theory, Methods \& Applications, vol. 75, no. 10, pp. 3973-3993, 2012.

[20] X. He, Y. A. Cui, and H. Hudzik, "The fixed point property of Orlicz sequence spaces equipped with the p-Amemiya norm," Fixed Point Theory and Applications, vol. 2013, article 340, pp. 1-18, 2013.

[21] Y. N. Ye, M. H. He, and R. Pluciennik, "P-convexity and reflexivity of Orlicz spaces," Commentationes Mathematicae Prace Matematyczne, vol. 31, pp. 203-216, 1991. 


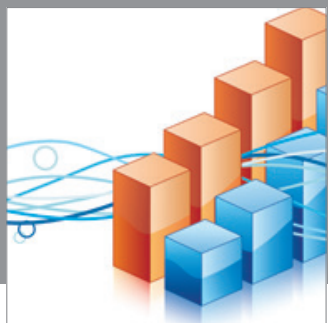

Advances in

Operations Research

mansans

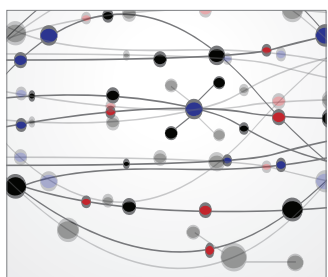

The Scientific World Journal
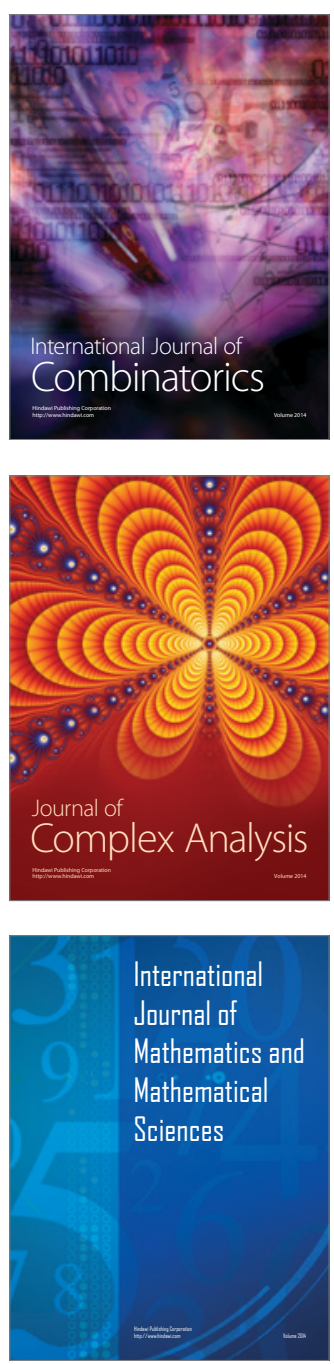
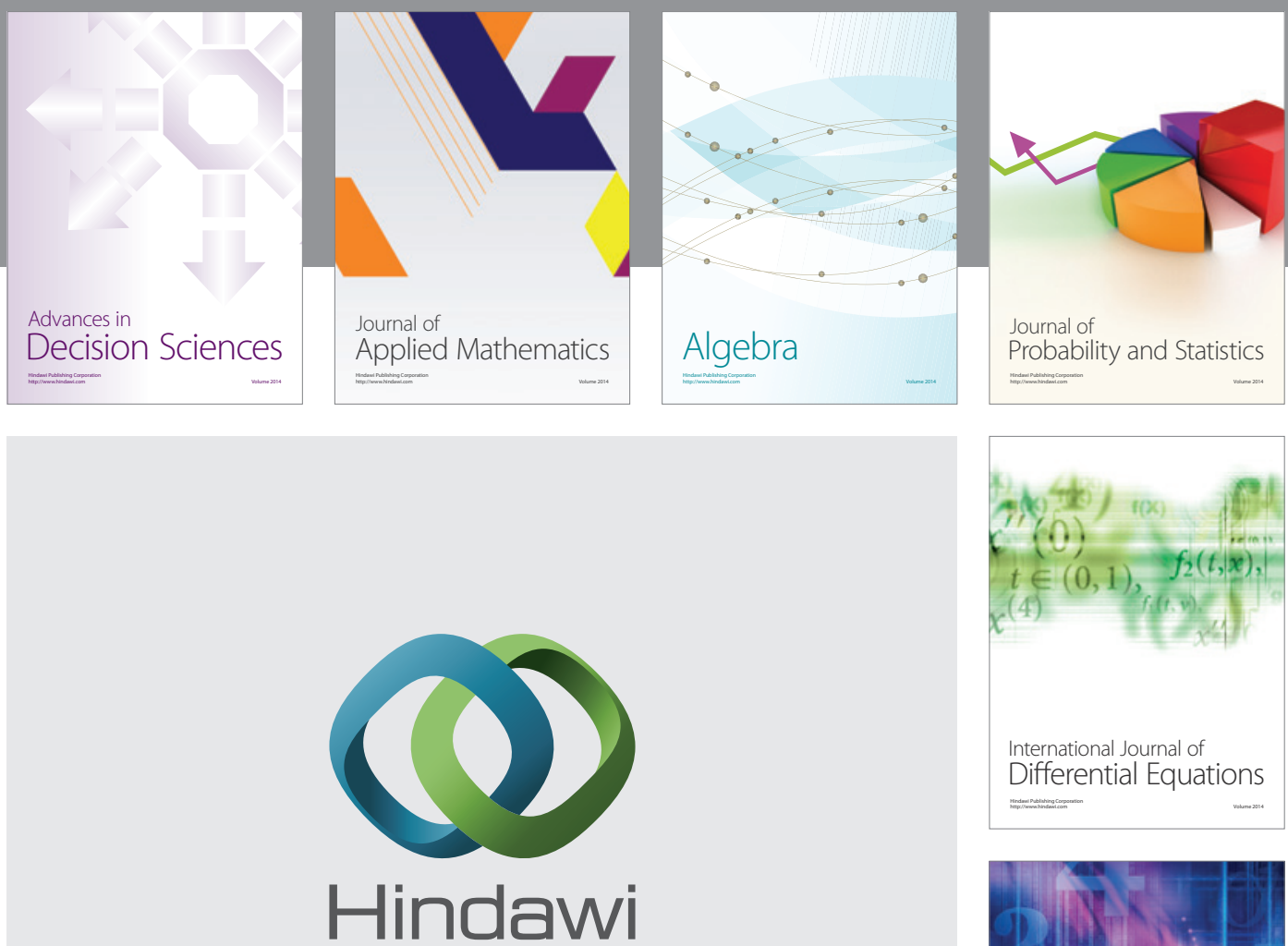

Submit your manuscripts at http://www.hindawi.com
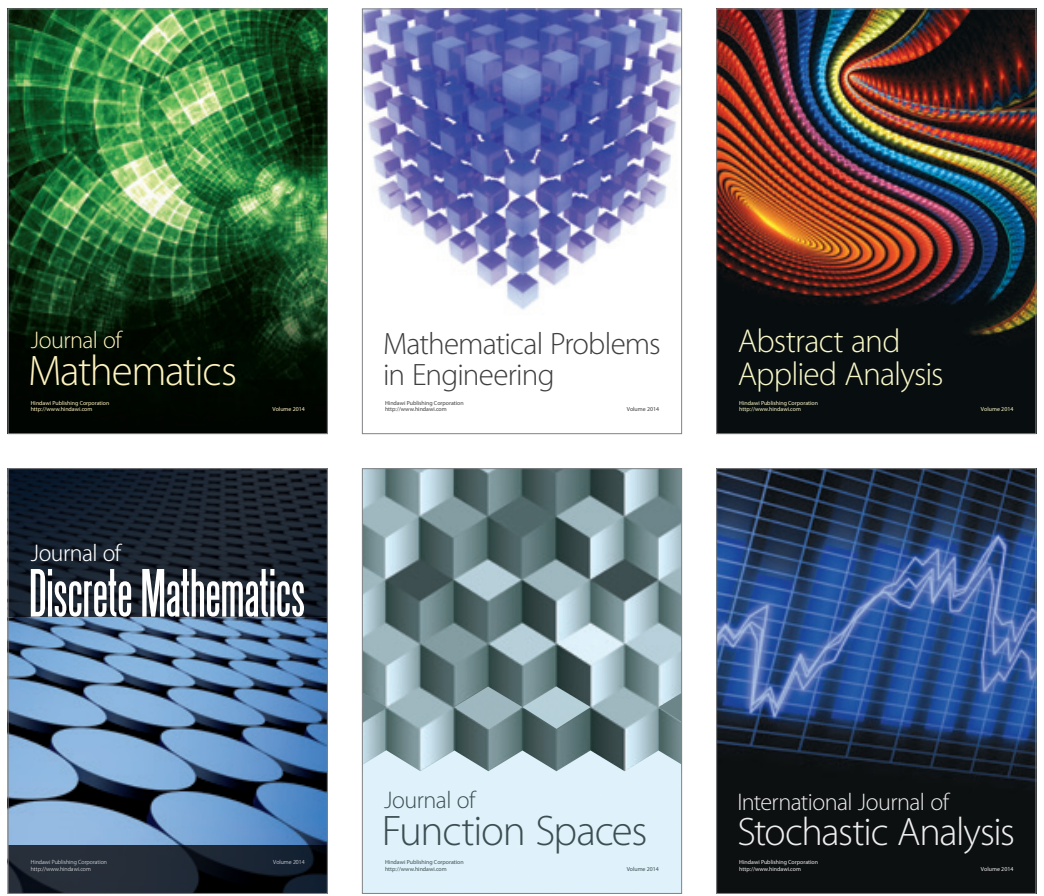

Journal of

Function Spaces

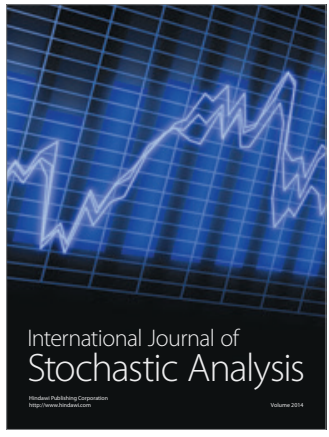

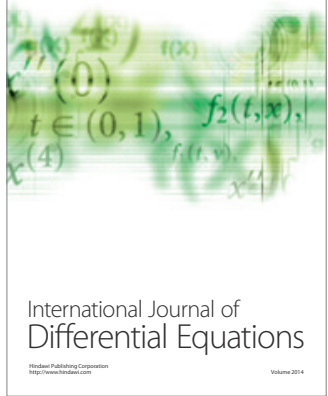
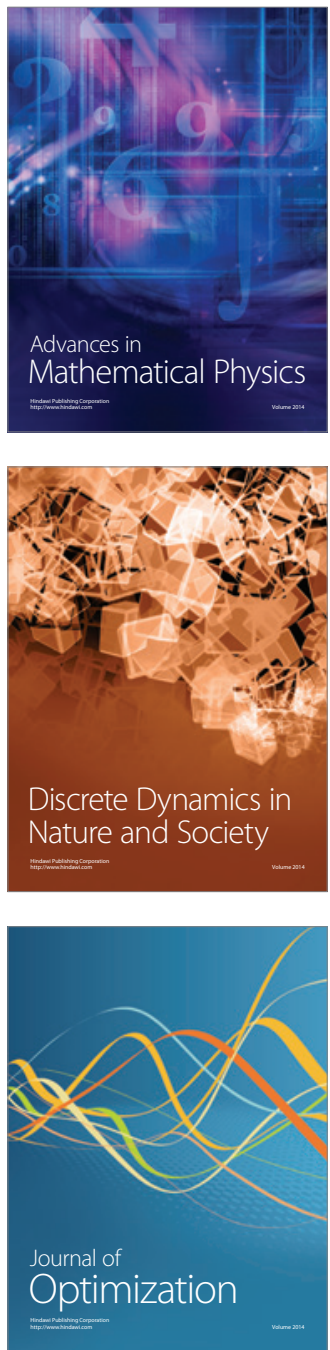